In 1986, Chevrie and Aicardi reviewed 184 cases of patients diagnosed with Aicardi syndrome and noted that others had described infantile spasms and typical lacunae in six girls whose corpus callosum appeared normal from a CT scan. ${ }^{2}$ Because magnetic resonance imaging (MRI) was not available to illustrate accurately the corpus callosum, they were not able to differentiate among a true mild expression of the syndrome (with partial agenesis of the corpus callosum), an incomplete form of the syndrome (normal corpus callosum), or a different syndrome altogether. The MR scan, in our case showing partial agenesis of the corpus callosum, confirms the diagnosis of a mild expression of Aicardi syndrome.

However, two questions arise. The first is whether the lesions, though mild and sparse, do in fact signal the presence of Aicardi syndrome. Our patient has been reviewed by several paediatric ophthalmologists; we are convinced that her lesions are a mild expression of the lesions characteristic of the syndrome. The second is the partial agenesis of the corpus callosum. The corpus callosum normally develops from the anterior to posterior direction. In our case, therefore, hypoplasia of the anterior corpus callosum with a normal posterior section, suggests that the corpus callosum initially developed and then regressed. However, there was no other evidence of focal cerebral atrophy. Since the exact nature of the corpus callosum malformation in the syndrome remains unclear, the atypical nature of our patient's corpus callosum does not rule out the diagnosis of Aicardi syndrome.

The prognosis is generally poor for children with the typical Aicardi syndrome. Although some degree of vision is present in most patients, psychomotor retardation is usually severe, and their mortality is probably high. However, follow up has been limited. Our patient would certainly be expected to have a much more favourable prognosis. Her biggest handicap appears to be her developmental delay of 4 to 5 years and her marked inattentiveness.

The genetic component of the disease will become an important issue should she have children of her own in the future, if she is indeed fertile. If Aicardi syndrome is an $\mathrm{X}$ linked dominant disease, as is thought to be the case, ${ }^{3}$ $50 \%$ of her offspring could be expected to carry the abnormal $\mathrm{X}$ chromosome. At present, genetic studies for our patient are not available, so we have no way of knowing whether she is an example of incomplete penetration or whether her genetic defect is an atypical one. We therefore cannot predict the effect of her disease on her offspring.

The triad of typical chorioretinal lacunae, infantile spasms, and hypoplasia of the corpus callosum in a girl with only mild developmental delay has led us to a diagnosis of a mild expression of Aicardi syndrome. It is important that ophthalmologists be aware of the existence of these mild cases since they are the ones which can secure the diagnosis by identifying the chorioretinal lesions pathognomonic of Aicardi syndrome.

This paper was prepared with the assistance of Medical Publications, The Hospital for Sick Children, Toronto, Ontario, Canada.

1 Aicardi J, Lefebvre J, Lerique-Koechlin A. A new syndrome: spasms in flexion, callosal agenesis, ocular abnormalities. Electroencephalogr Clin Neurophysiol 1965; 19: 609-10.

2 Chevrie JJ, Aicardi J. The Aicardi syndrome. In: Pedley TA, Meldrum BS, eds. Recent advances in epilepsy. Vol 3. Meldrum BS, eds. Recent advances in epilepsy.

3 Neidich JA, Nussbaum RL, Packer RJ, Emanuel BS, Puck JM Heterogeneity of clinical severity and molecular lesions in Aicardi syndrome. $\mathcal{F}$ Pediatr 1990; 116: 911-7.

\title{
Elevation of the right ptotic eyelid with clenching the teeth
}

\author{
Hiroko Kurome, Seiji Hayasaka
}

Department of
Ophthalmology, Shimane
Medical University,
Japan
H Kurome
S Hayasaka
Correspondence to:
Hiroko Kurome, MD,
Department of
Ophthalmology, Shimane
Medical University, Izumo,
Shimane, 693, Japan.
Accepted for publication
1 February 1994

The jaw-winking phenomenon of Marcus Gunn is typically seen as a retraction of one upper eyelid on opening the jaw or moving it to the side opposite the affected eye..$^{1-3}$ Elevation of a ptotic eyelid with clenching of the teeth, however, may be uncommon. ${ }^{3}$ We recently examined a patient with such a rare condition.

\section{Case report}

A 5-year-old girl was seen at our clinic with an unusual eyelid retraction in April 1993. The patient was born after an uneventful delivery on 6 August 1987. Her mother reported that the pregnancy also had been uneventful. The patient's paradoxical eyelid retraction was first noticed at age 7 months; the condition has appeared unchanged since then. She has been treated for atopic dermatitis since she was 4 years of age. No trauma to the face or eyes has been noted. Her family history was non-contributory.

On examination, the patient's visual acuity was 1.2 in both eyes. The eye positions were orthophoric, and the eye movements were 

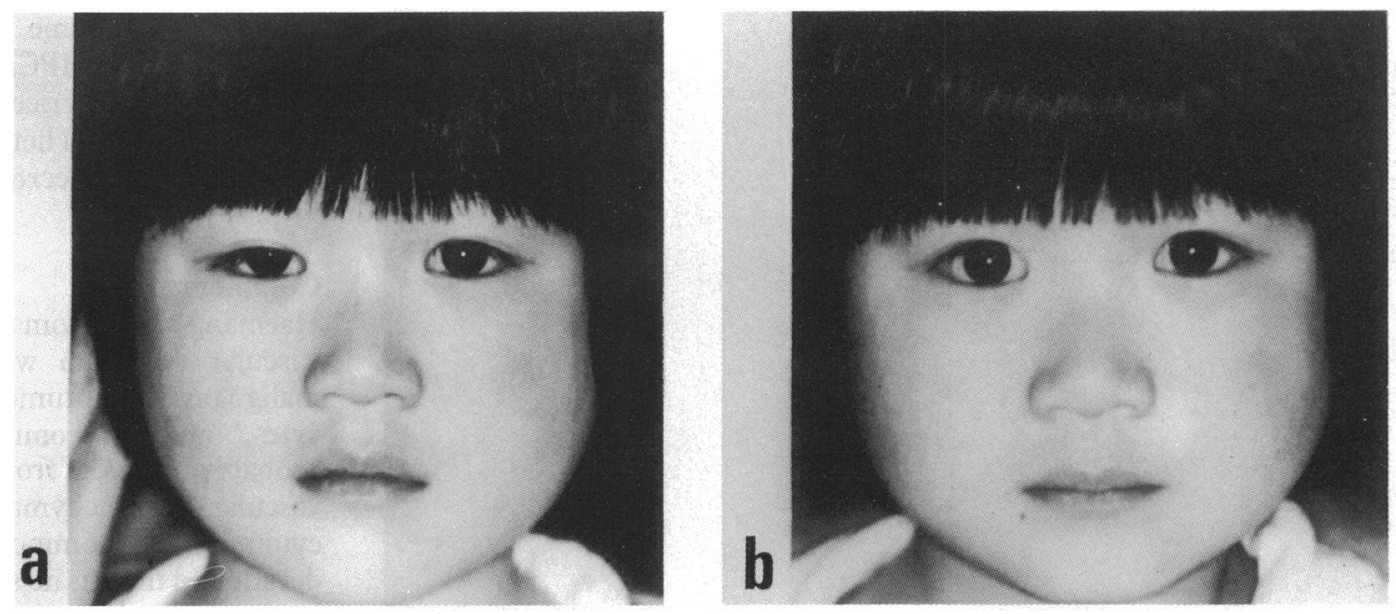

Figure 1 The patient's right upper eyelid, which usually is ptotic (a), elevates on clenching the teeth (b).

normal bilaterally. The right upper eyelid was ptotic (Fig la). No eyelid retraction was noted on opening the jaw or moving it to the left side. The right upper eyelid elevated on clenching the teeth (Fig 1b). The levator functions were normal bilaterally. The pupils were isocoric, and pupillary light reactions were prompt bilaterally. Both eyes appeared otherwise normal. Electroencephalography and computed tomography of the brain and orbits showed normal findings.

\section{Comment}

Marcus Gunn phenomenon is identified when the upper eyelid of one eye retracts on opening the jaw or moving it to the opposite side..$^{1-3}$ In our patient, the right ptotic eyelid elevated with clenching of the teeth. The movement seen in our patient was similar to those described in patients by Sano. ${ }^{3}$ Sano showed that electrical stimulation of the ipsilateral internal pterygoid muscle elicited elevation of the involved eyelid. We believe that elevation of the ptotic eyelid with clenching of the teeth, as found in our patient, may be rare.

We thank Dr T Setogawa for advice and Ms M A Gere for editorial assistance.

1 Duke-Elder S. Paradoxical lid-retraction. In: Duke-Elder S, ed, System of ophthalmology. Vol 12. St Louis, Mosby, 1972 ed, Systen

2 Miller NR. Normal and abnormal eyelid position and movement. In: Miller NR, ed. Walsh and Hoyt's clinical reuroophthalmology. 4th ed. Baltimore: Williams \& Wilkins, 1985 932-95.

3 Sano K. Trigemino-oculomotor synkineses. Neurologia 1959; 1 29-51.
Royal Eye Hospital, Oxford Road, Manchester M13 9WH S Sujatha R Sampath

R E Bonshek A B Tullo

Department of Pathological Sciences, University of Manchester, Oxford Road, Manchester M13 9PT

R E Bonshek

Correspondence to: Dr R E Bonshek. 26 January 1994

\title{
Conjunctival haemangiopericytoma
}

\author{
S Sujatha, R Sampath, R E Bonshek, A B Tullo
}

We report a case of conjunctival haemangiopericytoma (HPC) and discuss the clinical and pathological features and management of this tumour. Haemangiopericytoma most commonly arises in the lower limbs but is of interest to the ophthalmologist as a rare primary or secondary orbital neoplasm. ${ }^{1}$ Isolated cases of HPC arising within the lacrimal $\mathrm{sac}^{2}$ and choroid ${ }^{3}$ have been described. There are two reported cases of isolated conjunctival $\mathrm{HPC},{ }^{4}$ and HPC may involve the conjunctiva as an extension of orbital or eyelid neoplasm.

\section{Case report}

A 36-year-old white woman presented in May 1989 with a subconjunctival haemorrhage on the medial aspect of the right eye after sneezing.
She had noticed a small lump in the same area for several months (Fig 1). After 3 months, the clinical picture was unchanged and the possibility of a conjunctival vascular malformation or neoplasm was raised. The lesion was excised in September 1989. It was dissected free of a loose attachment to the medial rectus and the base was cauterised. An epithelial inclusion cyst was removed 1 month later. There have been no further problems to date.

Macroscopically the lesion measured $0.5 \times$ $0.4 \times 0.3 \mathrm{~cm}$, appeared well circumscribed, and was vascular. Histology showed an unencapsulated benign neoplasm, composed of plump spindle shaped and polygonal cells with indistinct cytoplasmic borders and plump spindle shaped and rounded nuclei. There were several irregular and partly collapsed endothelial lined vascular 